\title{
Recent Advances in the Performance and Understanding of the SNS ${ }^{*}$ Ion Source
}

\author{
RF Welton, MP Stockli, SN Murray and R Keller ${ }^{\#}$ \\ SNS, Oak Ridge National Laboratory, P.O. Box 2008, Oak Ridge, TN 37831, USA \\ ${ }^{\#}$ SNS, Lawrence Berkeley National Laboratory, 1 Cyclotron Rd.., Berkeley, CA, 94720, USA
}

\begin{abstract}
The ion source developed for the Spallation Neutron Source* (SNS) by Lawrence Berkeley National Laboratory (LBNL), is a radio frequency, multi-cusp source designed to produce $\sim 40 \mathrm{~mA}$ of $\mathrm{H}^{-}$with a normalized rms emittance of less than $0.2 \mathrm{pi} \mathrm{mm}$ mrad. To date, the source has been utilized in the commissioning of the SNS accelerator and has already demonstrated stable, satisfactory operation at beam currents of $\sim 30 \mathrm{~mA}$ with duty-factors of $\sim 0.1 \%$ for operational periods of several weeks. Once the SNS is fully operational in 2008, a beam current duty-factor of 6\% $(1 \mathrm{~ms}$ pulse length, $60 \mathrm{~Hz}$ repetition rate) will be required in order to inject the accelerator. To ascertain the capability of the source to deliver beams at this high duty-factor over sustained time periods, several experimental runs have been conducted, each $\sim 1$ week in length, in which the ion source was continuously operated on a dedicated test stand. The results of these tests are reported as well as a theory of the Cs release and transport processes which were derived from these data. The theory was then employed to develop a more effective source conditioning procedure as well as an improved Cs collar design. Initial results of tests employing a Cs collar with enhanced surface ionization geometry are also discussed.
\end{abstract}

\section{INTRODUCTION}

The Spallation Neutron Source (SNS) will be a large multinational user facility dedicated to the study of the dynamics and structure of materials by neutron scattering and is currently under construction at Oak Ridge National Laboratory (ORNL) ${ }^{1}$. Neutrons will be produced by directing $1 \mathrm{GeV}$ pulses of protons from a chain of linear accelerators and a compressor ring onto a liquid $\mathrm{Hg}$ target. In order to meet the baseline requirement of 1.4 MW of proton beam power on target, the ion source must produce $\sim 40$ $\mathrm{mA}$ of $\mathrm{H}$ within a 1-ms pulse at a repetition rate of $60 \mathrm{~Hz}$ (6\% duty-factor).

To date, the ion source has been extensively utilized in commissioning the SNS FrontEnd (FE) both at $\mathrm{LBNL}^{2}$ and ORNL ${ }^{3}$ as well as the Drift Tube Linac (DTL) ${ }^{4}$. In this role, the source has performed very well, with availability of the source and Low Energy Beam Transport (LEBT) reaching 98\% during the last commissioning period which spanned several weeks. During this time, the ion source was mainly operated at beam currents of $\sim 30 \mathrm{~mA}$ with a very low duty-factor of $\sim 0.1 \%$, although the design goal of 38 $\mathrm{mA}$ (6\% duty-factor) was briefly demonstrated both at LBNL and ORNL. On one occasion an endurance test was performed at LBNL in which the ion source was operated for $\sim 5$ days with a duty-factor of $2-3 \%$ delivering $\sim 25 \mathrm{~mA}$ of beam current.

*SNS is a collaboration of six US National Laboratories: Argonne National Laboratory (ANL), Brookhaven National Laboratory (BNL), Thomas Jefferson National Accelerator Facility (TJNAF), Los Alamos National Laboratory (LANL), Lawrence Berkeley National Laboratory (LBNL), and Oak Ridge National Laboratory (ORNL). SNS is managed by UT-Battelle, LLC, under contract DE-AC0500OR22725 for the US Department of Energy. 
Given the lack of performance data ${ }^{5}$ taken over long, sustained run-periods at beam duty-factors of $\sim 6 \%$, we are currently performing such tests on a dedicated test stand capable of unattended continuous operation. Two identical ion sources have been tested over the course of 8 experimental runs, each lasting approximately 1 week, while having performance and operational parameters electronically logged ${ }^{6}$. These data have then been used to develop a formulation of the Cs release and transfer process, which was then used to construct a more effective source conditioning procedure as well as guide the next iterations of SNS ion source development. Given the recent interest shown at this symposium in scaling of various types of $\mathrm{Cs}$ enhanced $\mathrm{H}$ ion sources to long-pulse, highduty-factor operation, the Cs transport model developed for the SNS ion source is described in sufficient detail to allow applicability to other $\mathrm{H}$ ion sources. Information gleaned from an initial test of the integrated Cs collar / outlet aperture, which employs an optimized ionization surface, is also discussed.

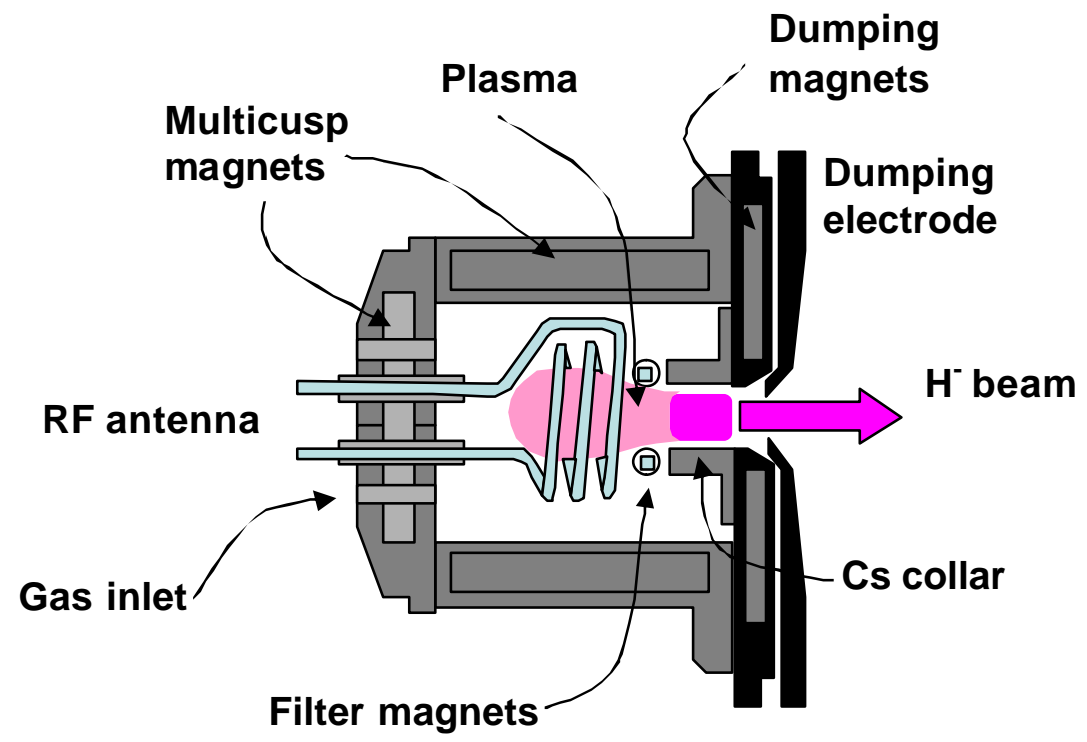

Figure 1. Schematic diagram of the SNS ion source.

\section{The Multicusp H Source and LEBT}

A schematic diagram of the $\mathrm{H}$ ion source is shown in Fig. 1. The source plasma is confined by a multicusp magnetic field created by a total of 20 samarium-cobalt magnets lining the cylindrical chamber wall and 4 magnets lining the back plate. Pulsed RF power ( $2 \mathrm{MHz}, 20-60 \mathrm{~kW}$ ) is applied to the antenna shown in the figure through a transformerbased impedance-matching network. The plasma is sustained between high-power RF pulses by continuous application of $\sim 200 \mathrm{~W}$ of $13.56 \mathrm{MHz}$ power to the same antenna. A magnetic dipole (150-300 Gauss) filter separates the main plasma from a smaller $\mathrm{H}^{-}$ generation region where low-energy electrons facilitate the production of large amounts of negative ions. An air heated/cooled collar, equipped with eight cesium dispensers, 
each containing a mixture of $\mathrm{Al}, \mathrm{Zr}$ and $\mathrm{Cs}_{2} \mathrm{CrO}_{4}$, surrounds this $\mathrm{H}$ generation volume. The RF antenna is made from copper tubing that is water cooled and coiled to $2 \frac{1}{2}$ turns and centered in the plasma chamber. A porcelain enamel layer insulates the plasma from the oscillating antenna potentials ${ }^{7}$. More details of this source design can be found in reference 8 .

Ions are extracted from the source by applying a continuous DC bias of $-65 \mathrm{kV}$ to the ion source and accelerating ions through a circular extractor electrode held at or near ground potential. The pulse structure of the ion beam is achieved by pulsing the $2 \mathrm{MHz}$ high-power RF creating the plasma. Closely coupled to the source is the Low Energy Beam Transport (LEBT) section of the SNS accelerator which matches the Twiss parameters of the extracted beam to that of the Radio Frequency Quadrupole, the first accelerator in the SNS chain. The LEBT, $10 \mathrm{~cm}$ in length, consists of two Einzel-type electrostatic lenses which can be independently voltage-controlled to provide optimal matching and is described in detail in Reference 2 and references therein. Identical LEBTs exist on the SNS front end as well as the ion source test stand which was employed in these investigations.

\section{High-Duty Factor Ion Source Tests - Runs 1-7}

Three nearly identical ion sources were shipped from LBNL to ORNL along with the front end system ${ }^{2,3}$. One source has been in nearly continuous service on the SNS accelerator serving to commission the machine. The other two sources have been employed in this study, each being run four times on the ion source test stand for a period of $\sim 1$ week (experimental Runs 1-8). The beam current was measured using a toroidal Beam Current Monitor (BCM) located a few cm downstream of the second Einzel lens of the LEBT and a Faraday cup located $\sim 20 \mathrm{~cm}$ downstream of the BCM. Prior to each run, the ion source was thoroughly cleaned using $15 \mu \mathrm{m}$ diamond-grit sandpaper, fresh Cs dispensers were installed and a new antenna was added. Each time a new source was mounted, the system was thoroughly leak-checked with a He leak detector.

Following the guidance of LBNL, the following ion source conditioning procedure was used for experimental Runs 1-7. The source was started with a low-duty-factor plasma of $\sim 0.1 \%$ achieved by pulsing $20-30 \mathrm{~kW}$ of the $2 \mathrm{MHz}$ RF power with a pulse width of $\sim 100 \mu \mathrm{s}$ and a repetition rate of $\sim 10 \mathrm{~Hz}$ for several hours. During this time the Cs collar was gradually heated to a nominal operating temperature of $\sim 300 \mathrm{C}$ by increasing the collar air temperature using an external air heater. Next, Cs was released into the source by raising the plasma duty-factor to $\sim 3 \%$ (pulse width: $\sim 1 \mathrm{~ms}$, repetition rate: $\sim 30 \mathrm{~Hz}$ ) and restricting the Cs collar air flow, allowing the collar to be heated to temperatures of $500-550 \mathrm{C}$ for $\sim 1 / 2$ hour. Finally, after 1 cesiation, the source was ramped to the nominal duty-factor of $7.3 \%$ used in these studies by increasing the RF pulse length to $1.2 \mathrm{~ms}$ and the repetition rate to $60 \mathrm{~Hz}$. It was necessary to exceed the beam dutyfactor requirement of $6 \%$ since the SNS will be unable to accelerate the initial peak due to excessive emittance in this region of the pulse. Once high-duty factor operation was established, the RF power was adjusted to give maximum beam current, typically 50-60 $\mathrm{kW}$. The Cesiation process described above was repeated as needed to keep the beam intensity as high as possible. The beam current was then sustained for $\sim 1$ week, long 
enough to resolve the beam current trend, unless the beam could not be increased above $20 \mathrm{~mA}$, in which case the run was terminated. After the source was run it was disassembled and inspected and, in each case, the source was found in generally the same condition as when installed with the exception of the Cs collar and RF antenna. The Cs collar was typically found discolored, noticeably black in appearance, with some evidence of sputtering. The condition of the antenna after each experimental run is given in the last column of Table I.

Figure 2 shows the electronically logged beam current for experimental Runs 5-7 measured with the BCM corrected for droop and averaged over the entire pulse. Similar beam current plots of experimental Runs 1-4 can be found in an earlier report ${ }^{9}$. The ion source downtime observed in Fig. 2 resulted from system trips or source instabilities which occurred during unattended periods. Had an operator been present on a 24-hour basis very little downtime would have been observed. The time of each source cesiation as well as changes in RF power and duty-factor are noted in the plot area of Fig. 2.

Experimental Run-5 is typical of each of the earlier tests (Runs 1-4) employing the original LBNL source configuration. The plot shows a maximum measured just after the source's initial cesiation followed by a steady beam attenuation rate ( $4 \mathrm{~mA} /$ day) which could only temporarily be abated with subsequent cesiations. In experimental Run- 6 , the downstream aperture of the Cs collar, shown later in Fig. 6, was removed to allow $\mathrm{H}$ ions produced to the collar surface to directly enter the ion beam.

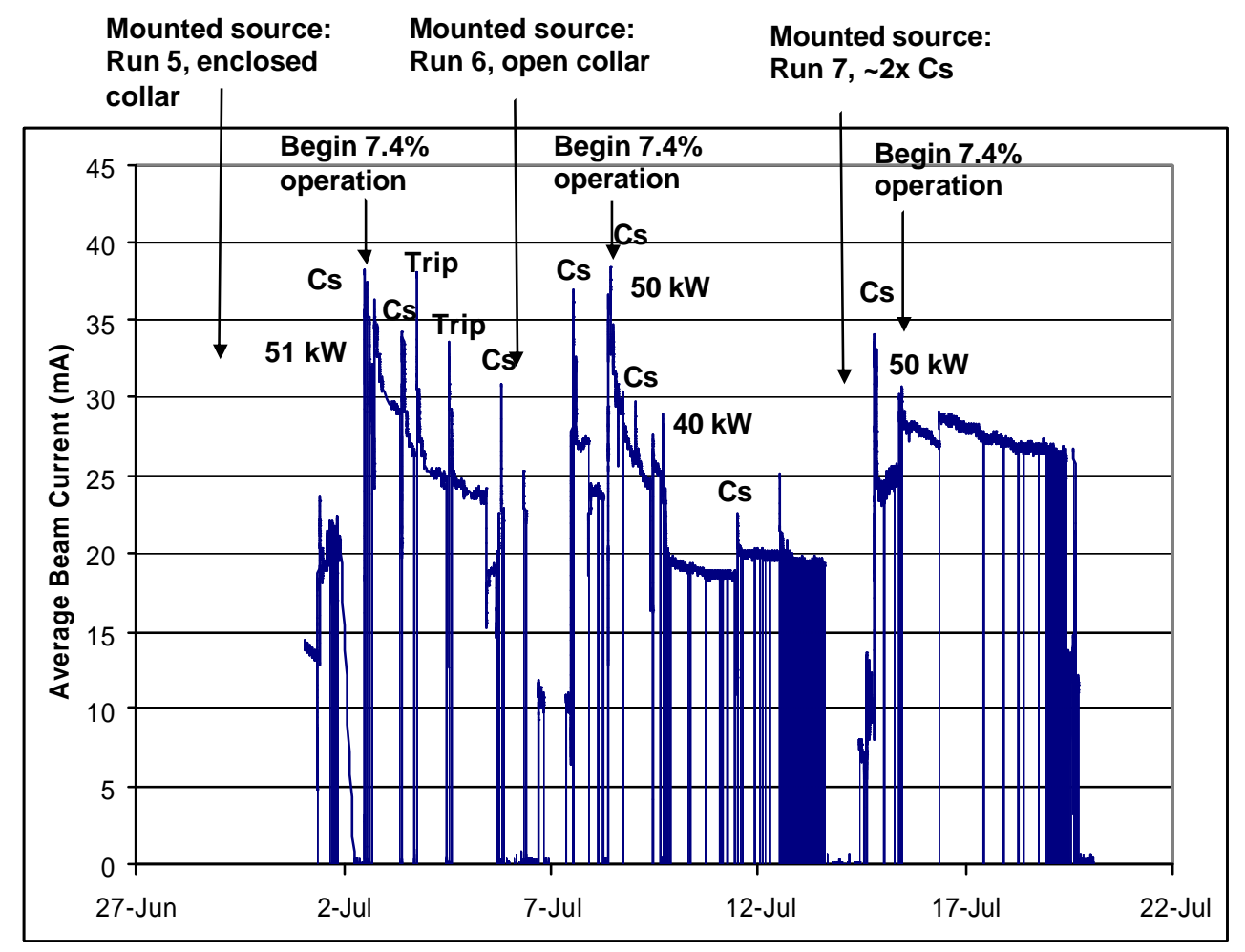

Figure 2. Measured beam current (averaged over the $1.2 \mathrm{~ms}$ pulse) produced from the source using the conditioning and cesiation procedures described above for experimental runs 5-7. 
This configuration required a higher $\mathrm{H}_{2}$ gas flow rate and resulted in a comparable initial beam intensity of $38 \mathrm{~mA}$ but a much greater beam attenuation rate of $9 \mathrm{~mA} /$ day. In experimental Run-7 additional Cs dispensers were added to the collar to approximately double the Cs supply. During this run the maximum beam current was somewhat less then earlier runs, but the beam attenuation rate of $2 \mathrm{~mA} /$ day was comparable to the best earlier runs.

Table I contains a statistical summary of each experimental run in which the source was operated with a $6 \%$ or higher duty-factor. Six percent duty-factor could not be reached in experimental Run-3 due to insufficient cooling of the LEBT extractor electrode and is therefore omitted from the table. The maximum beam current shown in the table is the largest current measured during the experimental run. The average beam current also shown in the table is computed by averaging all measured beam current values from the beginning of the run to termination excluding the down-periods which resulted from system trips. The power efficiency is computed from the average beam current divided by the $2 \mathrm{MHz}$ RF power used to generate the plasma during the run period. The run length, also shown, is defined as the total time the source ran with a duty-factor of $6 \%$ or greater which accounted for the majority of most run-periods. The average beam attenuation rate is determined by subtracting the maximum beam current achieved during the experimental run from the end-of-run beam current value and dividing by the time interval between these two points, shown as $\Delta \mathrm{t}$ in the table.

TABLE I. Statistical summary of the high-duty factor experimental runs.

\begin{tabular}{|c|c|c|c|c|c|c|c|c|c|c|}
\hline $\begin{array}{c}\text { Run } \\
\#\end{array}$ & Config. & $\begin{array}{c}\mathrm{RF} \\
\text { powe } \\
\mathrm{r} \\
(\mathrm{kW})\end{array}$ & $\begin{array}{l}\text { Max } \\
\text { current } \\
\text { (mA) }\end{array}$ & $\begin{array}{l}\text { Average } \\
\text { current } \\
(\mathrm{mA})\end{array}$ & $\begin{array}{c}\text { Power } \\
\text { Efficiency } \\
(\mathrm{mA} / \mathrm{kW})\end{array}$ & $\begin{array}{l}\text { Run } \\
\text { length } \\
\text { (days) }\end{array}$ & $\begin{array}{c}\text { \# of } \\
\text { Cesia- } \\
\text { tions }\end{array}$ & $\begin{array}{c}\text { Beam } \\
\text { attenuation } \\
(\mathrm{mA} / \mathrm{day})\end{array}$ & $\begin{array}{c}\Delta t \\
\text { days }\end{array}$ & $\begin{array}{l}\text { Antenna } \\
\text { condition }\end{array}$ \\
\hline 1 & Normal & 40 & 28 & 18 & 0.45 & 1.6 & 4 & 10 & 1.8 & $\begin{array}{l}\text { Punctures outside } \\
\text { plasma region / } \\
\text { light conductive } \\
\text { coating }\end{array}$ \\
\hline 2 & Normal & 40 & 30 & 25 & 0.63 & 8.0 & 6 & 1.5 & 4 & $\begin{array}{c}\text { Punctures in } \\
\text { plasma region / } \\
\text { light conductive } \\
\text { coating }\end{array}$ \\
\hline 4 & $\begin{array}{l}\text { Normal } \\
\text { (fwd } \\
\text { ant) }\end{array}$ & 60 & 30 & 28 & 0.47 & 4.1 & 4 & 2.3 & 3 & $\begin{array}{l}\text { No punctures / } \\
\text { light conductive } \\
\text { coating }\end{array}$ \\
\hline 5 & $\begin{array}{l}\text { Normal } \\
\text { (fwd } \\
\text { ant) }\end{array}$ & 51 & 38 & 28 & 0.55 & 3.5 & 2 & 4.6 & 3 & $\begin{array}{l}\text { No punctures / } \\
\text { light conductive } \\
\text { coating }\end{array}$ \\
\hline 6 & $\begin{array}{l}\text { Open } \\
\text { collar }\end{array}$ & 50 & 38 & 27 & 0.54 & 1.7 & 3 & 9 & 1.5 & $\begin{array}{l}\text { No punctures / } \\
\text { light conductive } \\
\text { coating }\end{array}$ \\
\hline 7 & $\sim 2 x \mathrm{Cs}$ & 50 & 34 & 27 & 0.56 & 4.5 & 1 & 2 & 5 & $\begin{array}{c}\text { No punctures / } \\
\text { heavy conductive } \\
\text { coating }\end{array}$ \\
\hline
\end{tabular}


Experimental Runs 1-5 show that using the original LBNL source configuration and conditioning procedure the source will, on average, initially produce $32 \mathrm{~mA}$ which immediately begins to decay at an average rate of $4 \mathrm{~mA} /$ day. We also note that 1 out of 7 antennas were found punctured in the plasma region. This clearly falls short of the SNS requirement of $\sim 40 \mathrm{~mA}$ sustained for $\sim 21$-day operational periods. In the following section we carefully examine these data and develop a qualitative model of Cs transport in order to guide design and procedural modifications to the source necessary to realize this goal.

\section{Data Analysis}

Since we have already shown that beam currents close to the SNS requirement can be produced only for short periods of time, this analysis will focus on determining the cause of the large observed beam attenuation rates. It is widely held that Cs-enhanced, multicusp ion sources produce $\mathrm{H}^{-}$ions both on plasma facing surfaces and in the plasma volume. It is also believed that the addition of Cs lowers the work function of the surface thereby enhancing the $\mathrm{H}^{-}$yield ${ }^{10}$. In our source, during the nominal cesiation procedure a maximum of $\sim 2.7 \times 10^{17} \mathrm{Cs}$ atoms are delivered to the source (see below) with the $\mathrm{H}^{-}$ enhancement persisting for several hours to several days. If Cs were to significantly partake in volume processes it would have to be present in such a high volume density it would be pumped from the source on much shorter time scales. In this analysis we therefore consider the Cs-enhancement to be purely a surface effect. Without the addition of Cs the SNS source will produce about 10-15 mA of $\mathrm{H}$ current. This can only be increased to $\sim 40 \mathrm{~mA}$ when $\mathrm{Cs}$ is added. We therefore consider the $25-30 \mathrm{~mA}$ of additional beam current to be entirely surface-produced.

We monitor the hydrogen spectral lines emitted from the plasma during each experimental run using a fiber-coupled optical spectrometer (300-1400 nm). Since the fiber is affixed in a stationary position to a plasma-viewing window we can observe changes in plasma density over time by monitoring changes in spectral intensities. We can also detect changes to the plasma temperature by monitoring the ratio of the hydrogen lines. Plasma impurities can be tracked by observing the intensity of the background peaks. Cs-lines cannot be observed since Cs resides mostly on the surface rather than in the plasma volume, as discussed above. During the experimental runs we see essentially no changes in the se plasma properties (at constant RF power) while observing considerable beam attenuation rates of $\sim 4 \mathrm{~mA} /$ day (see Fig. 2). Since the plasma conditions apparently remain the same, we can attribute most of the observed beam attenuation to a reduction in surface rather than volume production.

Theoretically, efficient surface ionization requires (i) an intense flux of ions and/or fast neutrals arriving at the surface from the plasma core (Kishinevskii probability increases rapidly with bombardment energy ${ }^{10}$ ), (ii) a coating of $\mathrm{Cs}$, on the order of a monolayer, creating a low work-function ionization surface and, (iii) efficient extraction of the $\mathrm{H}$ ions once they are produced. Since optical spectroscopy suggests plasma conditions are essentially unchanged during an experimental run, conditions $\mathrm{i}$ and iii likely remain unchanged. This leaves the most likely cause of the observed beam attenuation as failure 
to maintain a low work function surface. The apparent increase in work function cannot be attributed to impurity contamination since, as we will show later, the flux and energy of hydrogen ions from the plasma is sufficient to rapidly kinetically eject adsorbed Cs and other reactive impurities from the surface. Thus we conclude that the increase in work function most likely results from limitations in the Cs supply. We will now look closely at the two processes required to deliver Cs to the ionization surface: Cs release from the dispensers and subsequent transport to the ionization surface.

\section{The Kinetics and Thermochemistry of Cs Release}

Cs is introduced into the ion source using the commercially available alkali metal dispensers from SAES Getter Corporation ${ }^{11}$. Each source contains eight Cs cartridges (dimensions: $12 \times 1.4 \times 1 \mathrm{~mm}$ ) containing a compressed powder mixture of $17 \% \mathrm{Cs}_{2} \mathrm{CrO}_{4}$, $70 \% \mathrm{Al}$ and $13 \% \mathrm{Zr}$ with the later two species configured as intermetallic compounds forming the low-temperature getter ST-101 ${ }^{11}$. Each cartridge contains $5.2 \mathrm{mg}$ of elemental Cs. In order to determine the Cs production rate, several experiments were previously conducted in which the dispensers were heated and the quantity of released Cs was monitored using atomic absorption spectroscopy $(852.1 \mathrm{~nm})^{12}$. The Cs release rate determined from these measurements, scaled to 8 dispensers (the nominal Cs load in the ion source) is given by Eqn. 1 .

$$
\phi(\text { atoms } / s)=1.125 \times 10^{24} \exp (-18500 /(273+T(C)))
$$

The minimum flow-rate observed from a single dispenser cartridge was $7.5 \times 10^{13}$ atoms $/ \mathrm{s}$ measured in the 480-560 $\mathrm{C}$ range, depending on the particular dispenser sample.

Analysis of this mixture using the chemical thermodynamic equilibrium computer code $\mathrm{HSC}^{13}$ shows the following dominant reactions :

$$
\begin{aligned}
& 4 \mathrm{Cs}_{2} \mathrm{CrO}_{4}+5 \mathrm{Zr} \rightarrow 8 \mathrm{Cs}(\mathrm{g})+5 \mathrm{ZrO}_{2}+2 \mathrm{Cr}_{2} \mathrm{O}_{3} \\
& 6 \mathrm{Cs}_{2} \mathrm{CrO}_{4}+10 \mathrm{Al} \rightarrow 12 \mathrm{Cs}(\mathrm{g})+5 \mathrm{Al}_{2} \mathrm{O}_{3}+3 \mathrm{Cr}_{2} \mathrm{O}_{3}
\end{aligned}
$$

The thermodynamic equilibrium concentration of each species in the Cs dispenser subjected to an $\mathrm{H}_{2}$ atmosphere (held at the ion source pressure of $10 \mathrm{mTorr}$ ) was also computed by the code as a function of temperature and is shown in Fig. 3. We see that the formation of elemental $\mathrm{Cs}$ is thermodynamically favored at all temperatures shown, and above $200 \mathrm{C} \mathrm{Cs}$ vapor dominates over the condensed state. Running the same simulation without the ST-101 getter material (Al and Zr) reveals elemental $\mathrm{Cs}$ does not form at temperatures below $\sim 950 \mathrm{C}$ (as shown in Fig. 4). Thus, in the absence of the getter material ST-101 the $\mathrm{Cs}_{2} \mathrm{CrO}_{4}$ will not release $\mathrm{Cs}$ until nearly $1000 \mathrm{C}$. This suggests that we should be concerned about chemically reacting the ST-101 material with reactive gases present in the source - precisely what the gettering material is intended to do! 


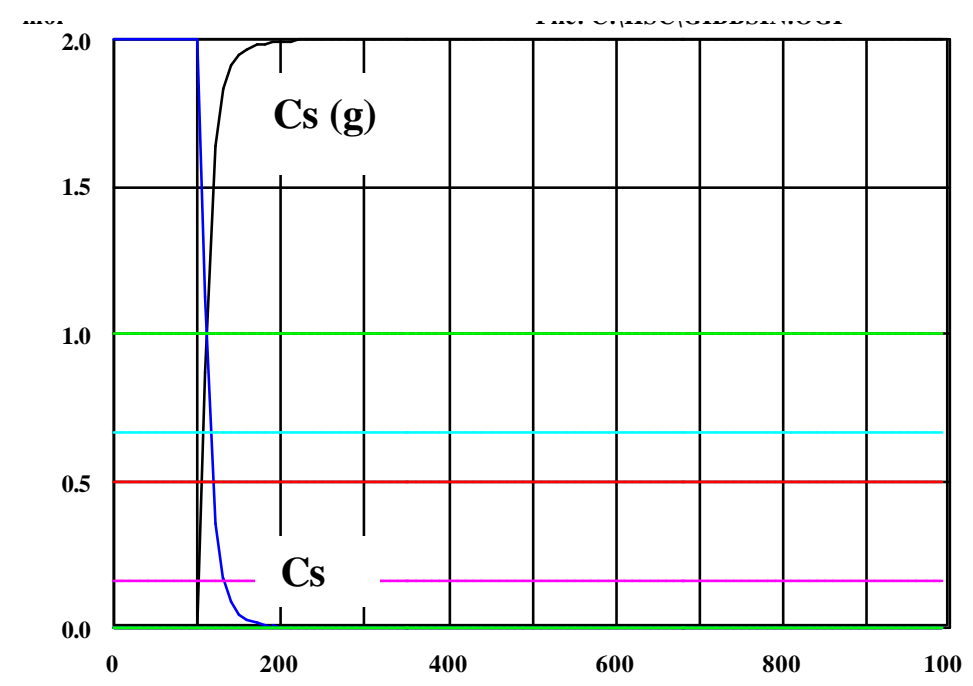

Figure 3. HSC calculation of the equilibrium concentrations of the components in the SAES Cs dispensers in $\mathrm{H}_{2}$ gas (10 mTorr). Note for simplicity hydrogen compounds are not plotted.

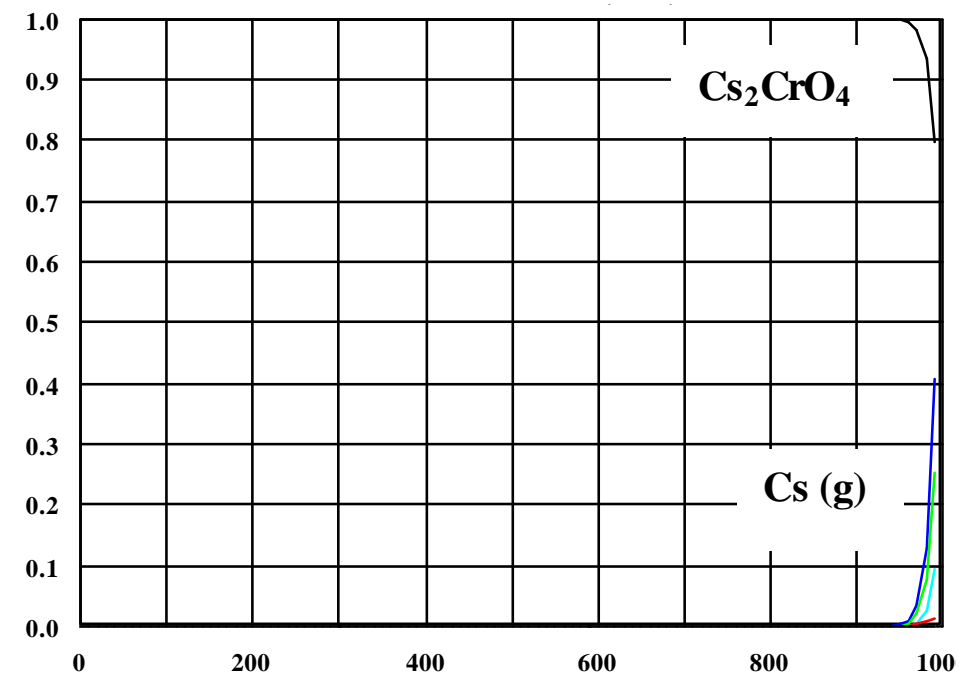

Figure 4. HSC calculation of the equilibrium concentrations of the components in the SAES Cs dispensers assuming the ST-101 getter is completely inactive. Note: for simplicity compounds containing hydrogen are not plotted.

The ST-101 product literature ${ }^{11}$ states that reactive gases such as $\mathrm{O}_{2}, \mathrm{CO}, \mathrm{CO}_{2}, \mathrm{H}_{2} \mathrm{O}$ and $\mathrm{H}_{2}$ begin to be sorbed by the getter material at temperatures about $250 \mathrm{C}$ and are readily sorbed at $300 \mathrm{C}$. The literature also states that opposed to the other reactive gases listed, $\mathrm{H}_{2}$ does not chemically react with the getter material, but instead forms a solid solution which has the characteristic of reversibility. Other reactive gasses are particularly harmful to the Cs dispenser since they are sorbed irreversibly by the ST-101 getter material and react with $\mathrm{Al}$ and $\mathrm{Zr}$ forming refractory compounds which inhibit $\mathrm{Cs}$ release. Unfortunately, analysis of the residual gas evolved by the source reveals that $\mathrm{O}_{2}, \mathrm{CO}$, $\mathrm{CO}_{2}$ and $\mathrm{H}_{2} \mathrm{O}$ are abundantly released during source outgassing each time the plasma 
chamber is heated to a higher temperature by increasing the average RF power (pulse RF power and duty-factor). Thus, care should be taken to outgas the source thoroughly at or above the desired operational duty-factor and power level while maintaining collar temperatures below $250 \mathrm{C}$.

Experience has shown that exposing the source to very small vacuum leaks while the Cs collar is at operating temperature not only immediately reduces the $\mathrm{H}$ beam intensity to pre-cesiation levels but also renders any subsequent cesiation attempts completely ineffective. The characteristic beam attenuation curve which occurs after the initial cesiation is shown in Fig. 2 for each source. Here an approximately exponential decay is observed. Subsequent cesiations only temporarily increase the beam current which rapidly decays, also in an exponential fashion, to the trend line set by the initial cesiation. Eventually, subsequent cesiations are completely ineffective at enhancing beam current. This behavior is consistent with the above picture of Cs release being limited by the chemical activity of the ST-101 getter material. As the ion source operates the evolved reactive gases are chemically gettered by the ST-101 material. This forms refractory compounds which decrease the amount of $\mathrm{Zr}$ and $\mathrm{Al}$ which are available for reducing the $\mathrm{Cs}_{2} \mathrm{CrO}_{4}$. Since most of the ST-101 getter material is available at the beginning of the experimental run, the cesiations undertaken then are most effective. As the run proceeds, cesiations become less effective due to the decreased quantity of the ST-101 material. Theoretically, each newly mounted-source contains enough $\mathrm{Cs}$ for 500 standard cesiations, but in practice only $\sim 5$ cesiations are effective at boosting the $\mathrm{H}^{-}$current.

\section{High-Duty Factor Ion Source Tests - Run-8}

In order to test the above picture of $\mathrm{Cs}$ release and determine if the average beam attenuation rate of $4 \mathrm{~mA} /$ day could be improved we implemented a new ion source conditioning technique during experimental Run-8. Unlike the operating procedure employed for experimental Runs 1-7, in the new technique the source was outgassed at $\sim 7 \%$ duty-factor and $50 \mathrm{~kW}$ of $\mathrm{RF}$ power for $\sim 1$ day while maintaining the collar temperature below $150 \mathrm{C}$. Source high voltage was off during this time to prevent excessive electron heating of the LEBT, which would have occurred if an uncesiated source was run at high beam duty-factor. Fig. 5 shows the beam current over the course of a 10 day run.

During the run the Cs collar was operated between 70-300 $\mathrm{C}$ and we observed the lowest beam attenuation rate of $1.1 \mathrm{~mA} /$ day. This led to our highest average beam current of any experimental run: $30 \mathrm{~mA}$, suggesting much more Cs was, in fact, delivered to the source. Unfortunately, an $\mathrm{H}$ current of $30 \mathrm{~mA}$ is still less beam than required for SNS operation, so we must now consider in detail the Cs transport process.

\section{Cs Transport to the Ionization Surface}

Once elemental gaseous $\mathrm{Cs}$ has been released by the dispensers it must migrate to the active ionization surfaces. Fig. 6 shows an enlarge ment of the Cs collar shown in Fig. 1. 


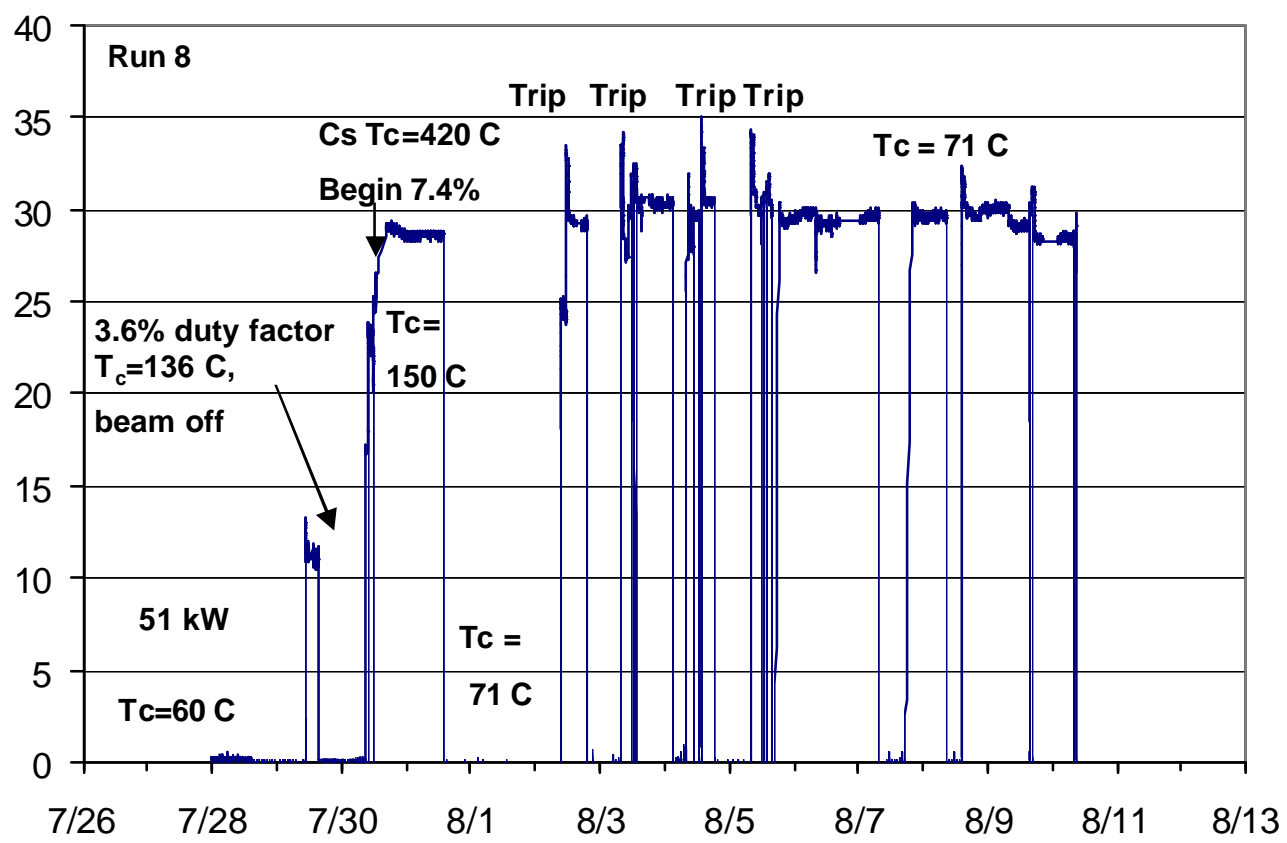

Figure 5. Measured beam current (averaged over the $1.2 \mathrm{~ms}$ pulse) during Run- 8 which employed the new source conditioning technique.

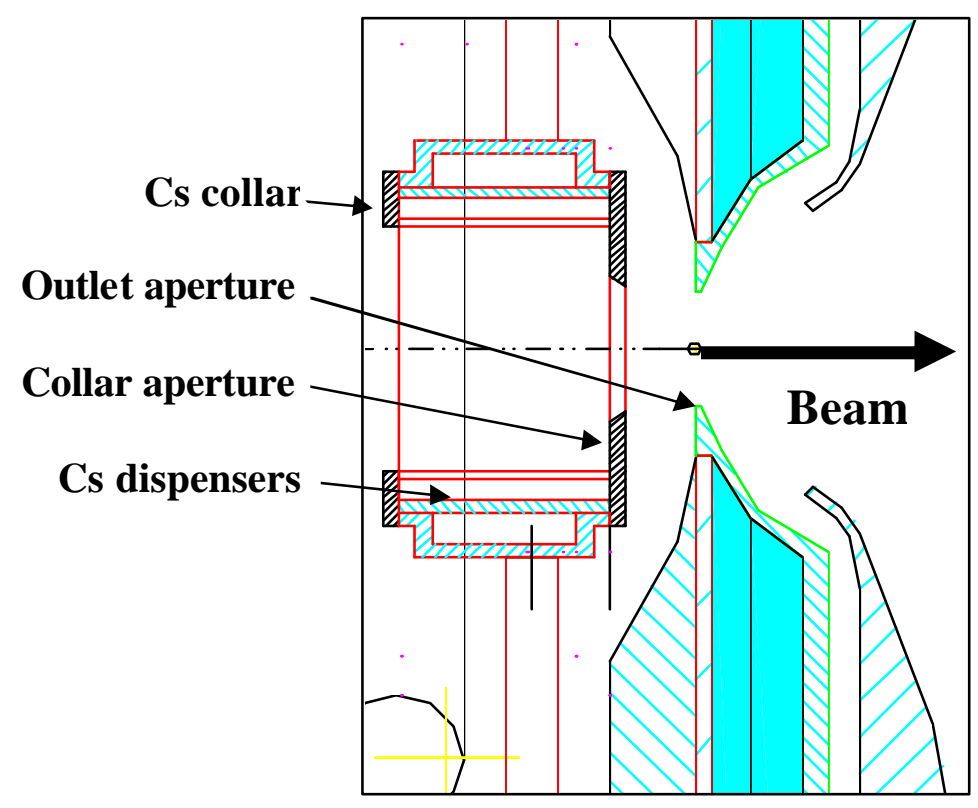

Figure 6. Close-up view of the standard Cs collar.

Before discussing the Cs transport process we must first clarify the location of the principal ionization surface(s). The NIETZSCHE Monte-Carlo code has been used to estimate the distance an $\mathrm{H}$ ion can travel without being destroyed by collisions with plasma particles. For discharges having parameters similar to the SNS ion source, calculation reveals $\sim 90 \%$ of $\mathrm{H}^{-}$ions having energies in the $1-10 \mathrm{eV}$ range will be destroyed after transiting the plasma a distance of $\sim 1 \mathrm{~cm}^{14}$. We therefore consider only 
surfaces within this short distance from the outlet of the source, which includes the interior surfaces of the Cs collar and outlet aperture.

During a typical cesiation the collar temperature is raised to $550 \mathrm{C}$. The extracted $\mathrm{H}$ beam typically shows no evidence of $\mathrm{Cs}$ enhancement while being held at this temperature. If the source has previously been cesiated and the collar is again raised to cesiation temperature, the beam current falls to levels of a completely uncesiated system until the temperature is lowered. It is unlikely that raising the temperature of the collar significantly affects the temperature of the outlet aperture since the latter is effectively water-cooled and radiant heat transfer is negligible in this temperature range. Thus we would expect the outlet aperture to be fully coated with Cs. Yet we see no enhancement strongly suggesting the outlet aperture plays only a minor role in surface production. In addition, Fig. 6 shows that most of the outlet aperture is shielded from direct plasma bombardment by the collar aperture. During Run-6 the Cs collar aperture was removed, and only then did we actually see beam enhancement while the collar was at cesiation temperatures. Therefore, we consider the plasma-facing surfaces of the Cs collar structure to be the primary ionization surface.

In order to elucidate Cs transport phenomena we must now estimate the rate at which Cs is lost from the collar in order to determine the rate at which the system needs to supply Cs. The bond strength $\mathrm{U}_{\mathrm{o}}$ between a metallic surface and a Cs ad-atom can be estimated by calculating the potential energy stored by the $\mathrm{Cs}^{+}$ion resting on the surface, separated from its image charge by 2 ionic radii $r_{o}$ summed with the energy lost by electron transfer to the surface (surface work function $\phi-C$ s ionization potential $I)^{15}$. This is expressed in Eqn. 4 and was evaluated to be $\mathrm{U}_{0} \sim 3.3 \mathrm{eV}$ under conditions of nearzero coverage for Cs on a metallic surface.

$$
U_{0}=\frac{e^{2}}{4 r_{0}}-(I-\phi)
$$

Under conditions of increasing Cs surface coverage $\theta$ (in units of mono-layers) the CsMe bond weakens and has been described empirically for the case of Cs ad-atoms on polycrystalline $\mathrm{W}^{15}$.

$$
U_{0}=\frac{2.78}{1+0.714 \theta}(e V)(0.06<\theta<0.6)
$$

Alternatively, others have measured the dependence of $\mathrm{U}_{0}$ for Cs coverage of monocrystalline $\mathrm{W}(110)^{16}$ and found

$$
U_{0}=3.37-2.78 \theta(e V)(0<\theta<1) .
$$


Under operational conditions the $\mathrm{Cs}$ coverage is adjusted for optimal $\mathrm{H}^{-}$production, which occurs when the work-function of the Cs-Me surface is minimal ${ }^{10}$. It is generally accepted that this condition occurs at $\theta \sim 0.5$, yielding $\mathrm{U}_{0}=2 \mathrm{eV}$ evaluated using either Eqn. 5 or $6^{17}$. The Frenkel equation can now be used to estimate the mean thermal lifetime of $\mathrm{Cs}$ chemisorbed on the surface at the operating temperature of $\sim 300 \mathrm{C}$, taking the enthalpy of adsorption to be $\Delta \mathrm{H} \sim \mathrm{U}_{0} \sim 2 \mathrm{eV}^{18}$.

$$
\tau \sim 6 \times 10^{-13} \exp \left(\frac{\Delta H}{k T}\right) \sim 63 \text { hours }
$$

Thus for an optimally filled monolayer Cs will thermally desorb from the surface as a result of evaporation at the rate of $\sim 10^{9} \mathrm{Cs} / \mathrm{s} / \mathrm{cm}^{2}$. We will shortly see that this loss rate is negligible compared with the $\mathrm{Cs}$ losses which result from kinetic ejection of ad-atoms from the surface as a result of ionic impact from plasma particles.

In the SNS ion source the $\mathrm{H}^{+}$ions primarily bombard the plasma facing surfaces of the Cs collar ejecting adsorped Cs. Fortunately, data exist characterizing positive ion beams extracted from RF-multicusp ion sources of virtually identical geometry to that of the SNS ion source and operated under essentially the same plasma conditions ${ }^{19}$. Beam current densities of $\sim 1.5 \mathrm{~A} / \mathrm{cm}^{2}$ or $10^{19}$ ions $/ \mathrm{s} / \mathrm{cm}^{2}$ of $\mathrm{H}^{+}$ions were observed from these sources at $\sim 50 \mathrm{~kW}$ of $\mathrm{RF}$ power. Other species such as $\mathrm{H}_{2}{ }^{+}, \mathrm{H}_{3}{ }^{+}$and $\mathrm{Cs}^{+}$are only observed as very small fractions of the primary $\mathrm{H}^{+}$ion beam. Beam energy distributions of the extracted protons have also been measured and were found to have a FWHM $=3.2$ $\mathrm{eV}$ for $0.5 \mathrm{~kW}$ of applied $\mathrm{RF}$ power and $3.6 \mathrm{eV}$ at $1 \mathrm{~kW}{ }^{20}$. Assuming a linear increase in the ionic energy spread with applied RF power, as suggested in reference 20, we would expect a FWHM $\sim 40 \mathrm{eV}$ at $50 \mathrm{~kW}$ of applied RF power. This corresponds to a Maxwellian distribution characterized by $\mathrm{kT}=17 \mathrm{eV}$ of bombarding $\mathrm{H}^{+}$ions striking the plasma-facing surfaces of the Cs collar.

The rate at which Cs is ejected from the metallic collar surface can be estimated using the approach of van Amersfoort ${ }^{18}$ which is based on the Bohdansky formula developed to calculate light ion, near-threshold sputtering yields of a variety of materials used in fusion research $^{21}$. In this approach, the Cs ejection rate was calculated by inputting the mass properties of the bulk metal target material along with the surface bond energy of the adatom, in our case, the Cs-Me bond energy of $\mathrm{Uo}=2 \mathrm{eV}$. According to this formulation the sputtering threshold energy $\mathrm{E}_{\mathrm{t}}$ is calculated using

$$
E_{t}=\frac{U_{0}}{\gamma(1-\gamma)}\left(m_{1}<0.3 m_{2}\right) \quad E_{t}=8 U_{o}\left(\frac{m_{1}}{m_{2}}\right)^{2 / 5}\left(m_{1}>0.3 m_{2}\right) \quad \gamma=\frac{4 m_{1} m_{2}}{\left(m_{1}+m_{2}\right)^{2}}
$$

Here $m_{1}$ and $m_{2}$ are the mass of the projectile and target, respectively. In our case the target material is 304 stainless steel which contains mainly $\mathrm{Fe}(\sim 72 \%), \mathrm{Ni}(\sim 9 \%)$ and $\mathrm{Cr}$ $(\sim 19 \%)$ which has an weighted average mass of $\mathrm{m}_{2}=55$. This yields a Cs-ejection threshold of $\mathrm{E}_{\mathrm{t}}=31 \mathrm{eV}$. The Bohdansky formula, which is valid for up to $\sim 20$ times threshold, is given as a function of projectile energy $\mathrm{E}^{21}$, 


$$
Y(E)=3.2 \times 10^{-3} m_{2}\left(\frac{4 m_{1} m_{2}}{\left(m_{1}+m_{2}\right)^{2}}\right)^{5 / 3} E^{1 / 4}\left(1-\frac{E_{t}}{E}\right)^{7 / 2} \text { atoms/ion }
$$

The numerical coefficient in Eqn. 9 has been modified to fit experimental sputtering yields measured for the specific case of $\mathrm{H}^{+}$bombardment of 304 stainless steel ${ }^{22}$. Folding the Maxwell-Boltsmann $\mathrm{H}^{+}$distribution into Eqn. 9 yields an average Cs ejection rate of $\sim 2 \times 10^{-4}$ atoms/ion for a Cs-filled monolayer.

We can now estimate the $\mathrm{Cs}$ ejection rate from a unit area $\left(1 \mathrm{~cm}^{2}\right)$ in the collar region of the source under conditions of optimal coverage: $10^{19} \mathrm{H}^{+} / \mathrm{s} \times 2 \times 10^{-4} \mathrm{Cs} / \mathrm{H}^{+} \times 0.07$ (plasma duty-factor) $\mathrm{x} 1 / 2$ monlayer $=\sim 7 \times 10^{13} \mathrm{Cs} / \mathrm{s}$. Comparing this loss rate to that of thermal desorption, calculated above, we see losses are dominated by surface sputterejection. Also we observe that at this ejection rate an optimally-filled monolayer would be depleted in $1 / 2 \times 3.56 \times 10^{14} \mathrm{Cs} / \mathrm{cm}^{2} / 7 \times 10^{13} \mathrm{Cs} / \mathrm{cm}^{2} \mathrm{~s} \sim 2.5 \mathrm{~s}$ if Cs were not continuously supplied to this surface! An unknown fraction of the sputter-ejected Cs must certainly return to the collar surface. Thus, we have shown that in order to maintain optimum coverage of the Cs collar, the system needs to continuously provide a flux of neutral Cs onto the surface $\sim 10^{14} \mathrm{Cs} / \mathrm{s} / \mathrm{cm}^{2}$.

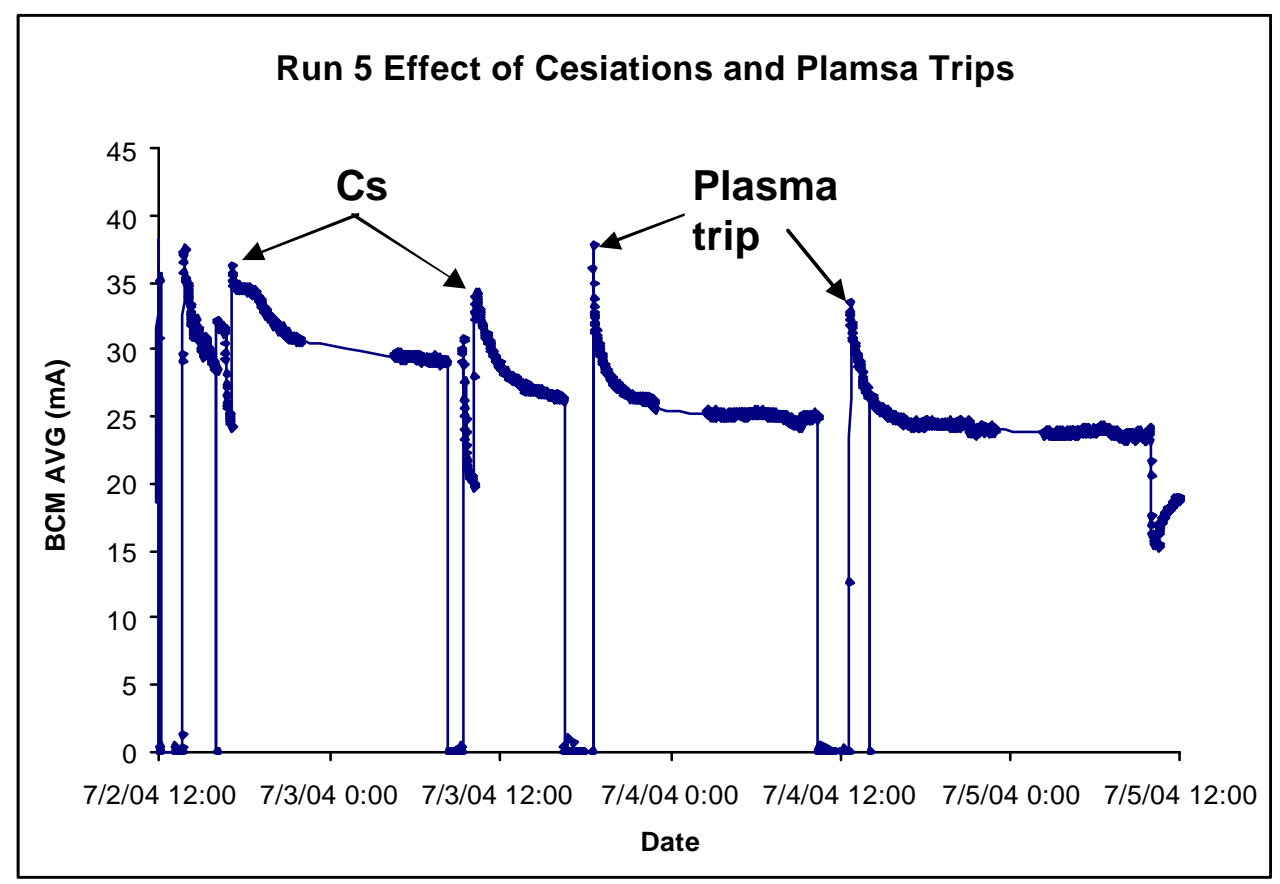

Figure 7. Detailed view of the $\mathrm{H}^{-}$beam current during Run-5 showing a characteristic response to cesiations and plasma outages.

Having described the location of the principal ionization surface in the source as well as the rate in which Cs needs to be delivered to that surface, we can now discuss the 
process of Cs transport between the dispenser and ionization surface. During a normal cesiation a maximum of $2.7 \times 10^{17} \mathrm{Cs}$ atoms leave the dispensers. Approximately $25 \%$ of the released Cs is immediately lost fom the source through the outlet aperture as a result of the close proximity of the dispensers to the outlet aperture. The balance of the released $\mathrm{Cs}$ enters the source and accumulates in cool areas. The accumulation rate depends on the difference between condensation rate, determined from the density of Cs atoms in the volume, and the evaporation rate, which can be determined from known Cs vapor pressure versus temperature curves. During this process Cs condensing on plasmafacing surfaces will likely be rapidly sputter-ejected and will accumulate in "shady regions" of the source. Most evidence of accumulated Cs was found behind the heat shield of the outlet aperture. After cesiation is complete and the collar temperature is lowered to the nominal operating temperature of $\sim 300 \mathrm{C}$, Cs flux from the dispensers is greatly reduced and $\mathrm{Cs}$ is now mainly supplied to the collar from the quantity which has accumulated within the source interior. As this 'dose' of Cs which was supplied to the source during cesiation diminishes the rate at which the ionization surface is fed Cs also diminishes. As the Cs feed-rate is reduced it becomes insufficient to maintain an optimal coating on the ionization surface and the $\mathrm{H}^{-}$beam current begins to attenuate.

In order to determine the relative importance of the direct Cs transport pathway (dispensers ? ionization surface) versus the indirect Cs transfer pathway (dispensers ? source body ? ionization surface) a specific experiment was performed. During Run-8, after an initial cesiation, the Cs collar was maintained at a constant temperature of $\sim 70 \mathrm{C}$ for the $\sim 10$ day run. Fig. 5 shows that our best $\mathrm{Cs}$-enhanced $\mathrm{H}^{-}$yield was maintained over that entire period with very small beam attenuation. Since the maximum Cs feedrate from the dispensers at the temperature of $70 \mathrm{C}$ could have only been $\sim 2$ atoms $/ \mathrm{s}$, as calculated by Eqn. 1, we conclude that the role of direct Cs transfer is small compared with the indirect process in the present source configuration. At the end of the run, the collar temperature was raised to the nominal $300 \mathrm{C}$ and little change in the beam current was observed.

Fig. 7 shows a detailed view of the beam response to source cesiations and plasma outages (trips) during Run-5. The approximately exponential character of these beam attenuation curves is consistent with this physical picture of indirect Cs transport: during a cesiation a finite amount of $\mathrm{Cs}$ atoms are introduced into the source and the rate at which these atoms re-evaporate $\Gamma$ is proportional ( $\mathrm{k}=$ proportionality constant) to the number of remaining condensed Cs atoms $n$.

$$
\frac{d n(\text { surface })}{d t}=-k \times n(\text { surface }) \rightarrow n(t)=\exp (-k \cdot t) \rightarrow \Gamma=k \exp (-k \cdot t)
$$

The approximately exponential beam decay also observed immediately after plasma outages (trips) is shown in Fig. 7 and can also be understood within the context of this physical picture. During the trip the entire ion source body cools with the exception of the Cs collar which is heated by an external air heating system. Cs continues to be fed to the source at a very small rate $\left(\sim 10^{10} \mathrm{Cs} / \mathrm{s}\right.$ at $\left.300 \mathrm{C}\right)$, accumulating on the cool source interior. Once the plasma is restored, the chamber heats and Cs is fed to the ionization 
surface at elevated rates resulting in conditions of more favorable coverage which accounts for the observed beam enhancement. Since the accumulated Cs is limited, the beam decays in approximately the same exponential fashion as it would after a standard cesiation described by Eqn. 10. Figure 2 also shows approximately exponential beam attenuation curves over the course of entire experimental runs, which is also a consequence of indirect Cs transport. In Run- 6 the attenuation is larger since Cs leaves the source faster due to removal of the Cs collar aperture. In Run-7 the attenuation was slower due to an increased supply of Cs.

Thus we have shown that in the current configuration of the SNS source indirect Cs transport plays a dominant role. It is also likely that many of the $\mathrm{H}$ ion sources used in fusion research that were discussed at this conference also operate in this fashion. There are a number of fundamental disadvantages to indirect Cs transfer: (i) During source operation the flow-rate of $\mathrm{Cs}$ evaporated from the source walls is essentially uncontrollable, dependent on the temperature distribution of the source and its construction details. (ii) Because the flow-rate is uncontrollable it cannot be well matched to the Cs loss rate from the ionization surface in order to insure optimal coverage. (iii) Cs transfer is also inefficient; most of the Cs will condense in regions which are too cold to re-evaporate at significant rates. A new collar for the SNS source is proposed in which $\mathrm{Cs}$ is directly transferred from the dispensers to the ionization surface.

\section{A Direct-Transfer Cs Collar}

In the previous section it was shown that indirect Cs transfer is inherently inefficient and almost certainly will feed Cs to the ionization surface at rates that are less than optimal. One simple solution to this problem is to insert a cooled/heated ionization surface immediately adjacent to the outlet aperture and independently control both Cs dispenser and ionization surface temperatures. These two structures need to be closely coupled to allow efficient particle transfer while, to the highest degree possible, being thermally isolated from each other. The proposed design of the reentrant ionization surface is shown in Fig. 8 and was developed using heat transfer software ${ }^{23}$.

The design features two air heating/cooling circuits which are independently controlled through two identical interfaces and allow operation of each component over an independent temperature range of 20-600 C. The existing Cs collar is now utilized purely as a Cs dispenser which is temperature-controlled using the existing system. The ionization surface has been re-located closer to the outlet aperture and has been inclined $10^{\circ}$ toward the plasma to receive a greater flux of particles from the plasma core. Thermal calculations show that a temperature uniformity of $\Delta \sim 10 \mathrm{C}$ under maximum plasma heating conditions can be achieved over the ionization surface. The entire assembly is supported by a Ta holder to provide protection from sputter damage, which has been observed on the existing stainless steel collar, as well as thermal isolation. Vacuum calculations suggest that approximately $70 \%$ of the Cs released from the dispensers will enter the surface ionization region. The design also allows easily interchangeable ionization surfaces to facilitate testing of different materials such as $\mathrm{Al}$ and Ta which could have favorable ionization characteristics. Higher work function materials are of interest since they bond more strongly to the Cs as well as yielding a 
lower effective work function when cesiated ${ }^{17}$. The system has currently been designed and will be tested within the next year.

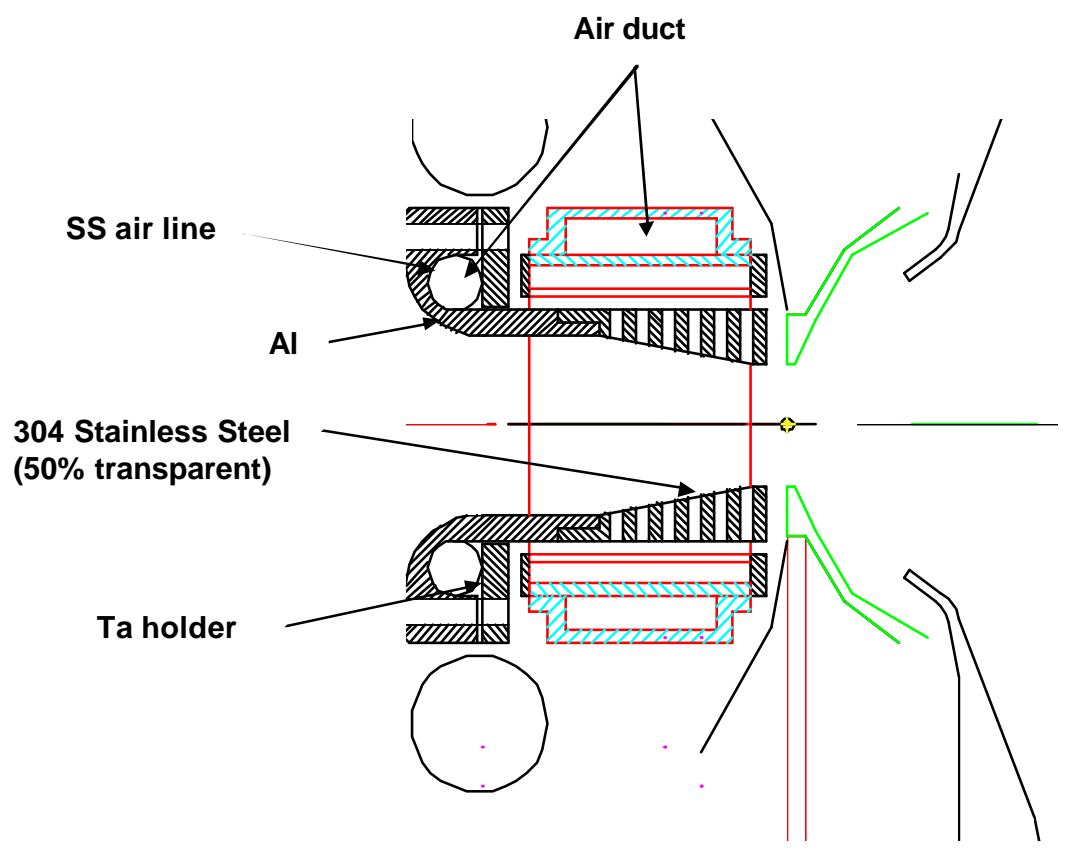

Figure 8. The design of the direct-transfer Cs collar.

\section{Initial Tests of the Integrated Cs Collar / Outlet Aperture}

The integrated Cs collar/outlet aperture has been conceived and developed as a result of collaborative ion source R\&D work undertaken between $\mathrm{LBNL}^{24}$ and ORNL ${ }^{14}$ and will be described in more detail in subsequent publications. Figure 9 shows a schematic view of the assembly. In this work, initial tests of the system are reported in order to explore the benefits of inclining the ionization surface towards the plasma and decreasing the distance from the surface to the outlet aperture, both features of the design of the direct-transfer Cs collar. The integrated Cs collar / outlet aperture also features the capability of applying a potential bias to the collar in order explore the $\mathrm{H}^{-}$enhancement which has been observed in the DESY multicusp source ${ }^{25}$.

Fig. 10 shows a comparison between the $\mathrm{H}^{-}$beam current extracted from the standard collar configuration shown in Fig. 6, and the integrated Cs collar / outlet aperture plotted as a function of applied RF power. The dramatically improved $\mathrm{H}^{-}$yield is most likely a consequence of the improved location and orientation of the ionization surface. This increase in performance seems to have come at the price of higher emittance. Fig. 11 shows the first emittance scan from this source configuration. Note the broad shoulders in addition to the usual bright center of the scan. The entire distribution has an RMS normalized emittance value of $0.35 \pi \mathrm{mm}$ mrad at $60 \mathrm{~mA}$ which is larger than emittances measured from the standard Cs collar at lower current levels of $40 \mathrm{~mA}$. These shoulders disappear when the source is in an uncesiated state, suggesting that the surface-produced $\mathrm{H}^{-}$is entering the beam with large energy and angular distributions as is typically seen 
with sputter ejected particles ${ }^{10,15}$. We also biased the collar to $-5 \mathrm{~V}$ with respect to the plasma, drawing 35 A of steady state discharge current and found essentially no enhancement of $\mathrm{H}$ yield. This result contrasts with DESY data which show significant beam enhancement in this range of voltages and currents, emphasizing the differences between the Cs-enhanced SNS ion source and the Cs-free, presumably pure volume, DESY ion source ${ }^{25}$.

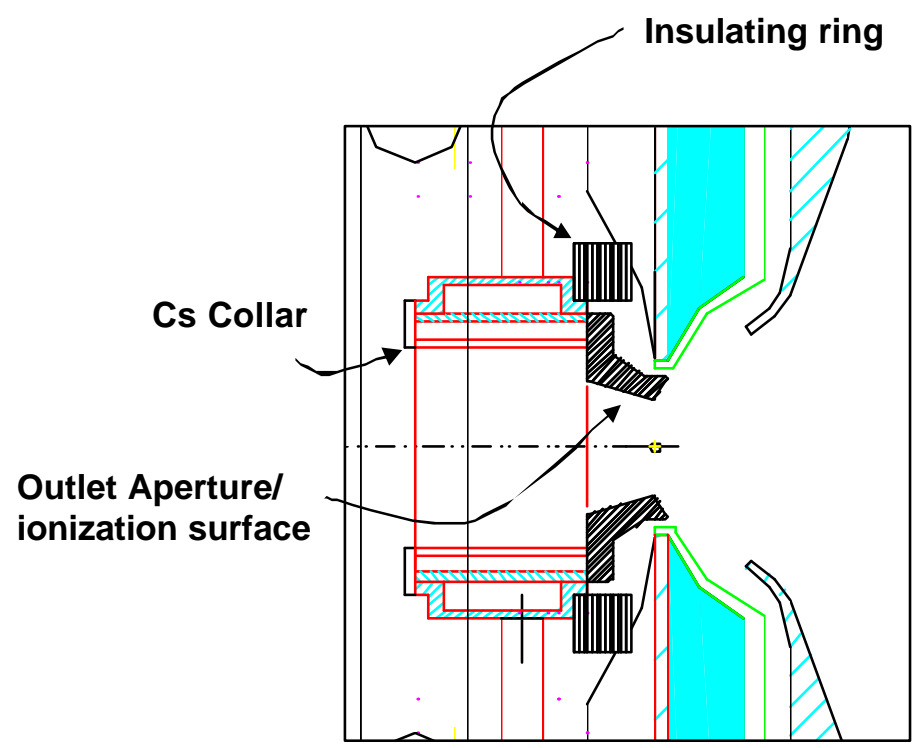

Figure 9. The Integrated Cs collar/outlet aperture.

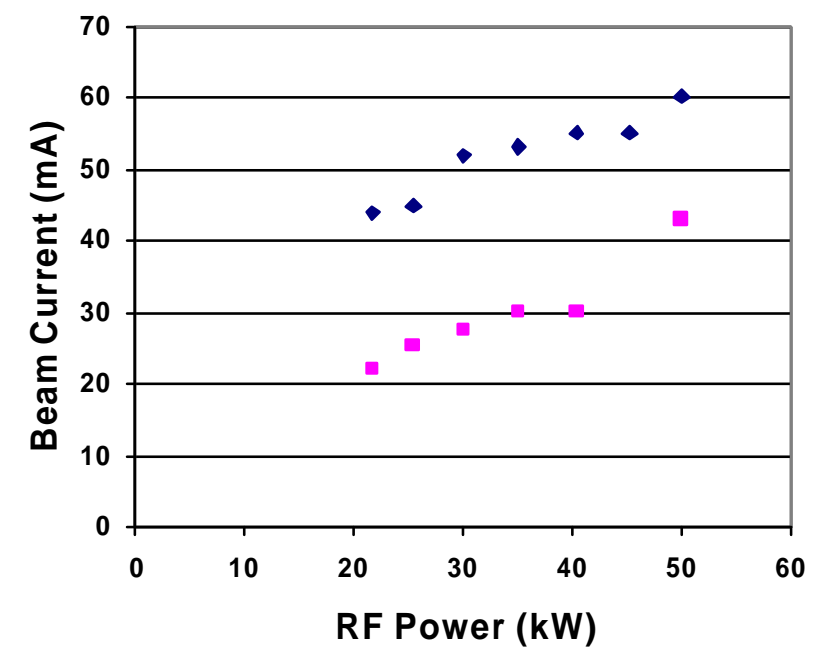

Figure 10. Beam current extracted from the integrated Cs collar/outlet aperture (diamonds) and from the standard source configuration (squares) versus applied RF power after 1 cesiation at full duty-factor.

Overall these results are very encouraging and show that prospects for improved $\mathrm{H}^{-}$yield from the geometrical improvements to the direct-transfer collar are quite strong. As 
discussed above, the direct-transfer collar design also features an ionization surface which is inclined toward the plasma, as well as a reduced distance to the outlet aperture as compared with the original design. Compared to the integrated Cs collar / outlet aperture the ionization surface is located several $\mathrm{mm}$ further away from the outlet aperture in hopes that resonant charge exchange will effectively cool these ions before entering the beam as apparently occurs in the present design.

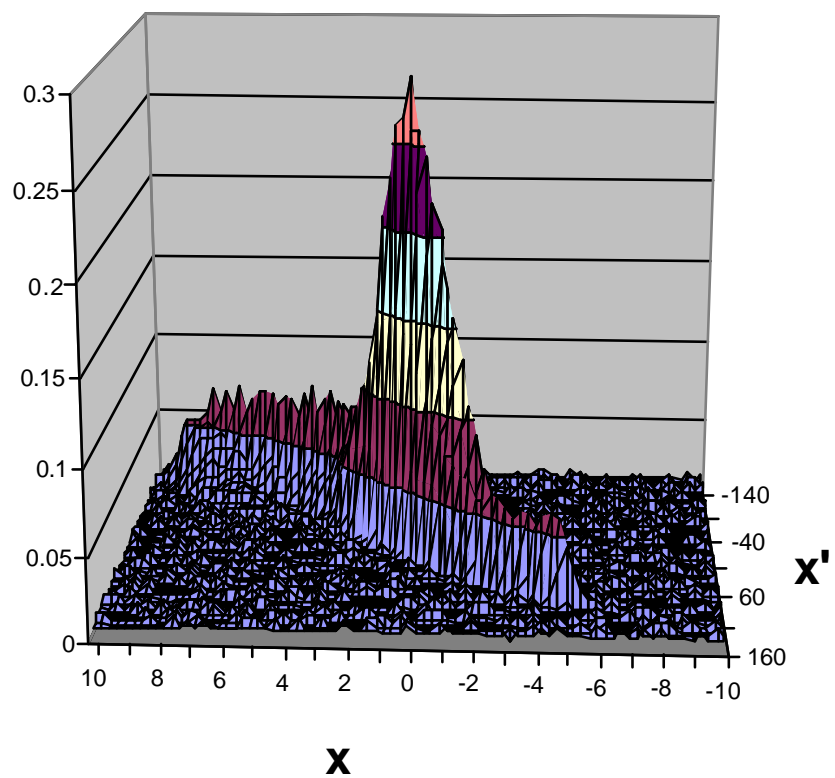

Figure 11. Emittance plot from the Integrated Cs collar/outlet aperture taken at $60 \mathrm{~mA}$. Note the presence of 'shoulders' at the base of the bright core.

\section{References}

1. N. Holtkamp, "Status of the SNS Project", PAC'03, Portland, USA, p. 11 ID: MOAL003.

2. R. Keller, et al., "Commissioning of the SNS Front End Systems at Berkeley Lab", EPAC'02, Paris, France, p. 1025, ID: THPLE012.

3. A. Aleksandrov, "Commissioning of SNS Front End Systems at ORNL," PAC'03, Portland, USA, p. 65, ID: MOPB002.

4. S. Henderson et al., "SNS Beam Commissioning Status", EPAC'04, p. 1255, ID: TUPLT168

5. R.F. Welton et al., "Development and Status of the SNS Ion Source", PAC'03 p. 3306 ID: FPAB009

6. R.F. Welton et al., Rev. Sci. Instrum. 75 (2004) 1793.

7. R.F. Welton et al., Rev. Sci. Instrum. 73 (2002) 1008.

8. R. Keller et al., Rev. Sci. Intrum. 73 (2002) 914.

9. R.F. Welton, et al. EPAC'04, Lucerne, France, ID: TUPLT175

10. M.E. Kishinevskii, Sov. Phys. Tech. Phys. 20 (1976) 799

11. SAES Getters S.p.A. Via Gallarate, 21520151 Milano, Italy

12. M. Succi, et al., Vaccum 35 (1985) 579

13. The HSC computer code, Outokumpu Research Oy, PO Box 60 FIN-28101 PORI, Finland

14. R.F. Welton, et al. EPAC'02, Paris, France, p.635 ID: THPLE019.

15. M. Kaminsky, "Atomic and Ionic Impact Phenomena on Metal Surfaces", Springer, New York, 1965

16. L.K. Hansen, "Thermionic Converters and Low Temperature Plasma", Techn. Inform. Center / US-

DOE-tr-1 (1978) 
17. G. Alton, Surface Sci. 175 (1986) 226.

18. P.W. Van Amersfoot, et al., J. Appl. Phys. 58 (1985) 2317

19. KN Lueng, et al. Nucl. Instrum. And Meth. B74 (1993) 291

20. Y. Lee, et al., Rev. Sci. Instrum. 68 (1997) 1398

21. J. Bohdansky, et al., J. Appl. Phys. 51 (1980) 2861

22. J. Bohdansky, et al., Proc. $7^{\text {th }}$ Int. Vacuum Congress and $3^{\text {rd }}$ Int. Conf. on Solid Surfaces, ed R.

Dobrozemsky, Wien, 1977.

23. Tera Analysis Ltd, Knasterhovvej 21, DK-5700 Svendborg, Denmark

24. R. Keller, et al, "Design, Operational Experiences and Beam Results Obtained with the SNS H- Ion Source and LEBT at Berkeley Lab," AIP Conf. Proc. 639, pp. 47-60 American Institute of Physics,

Melville, NY (2002)

25. J. Peters, Rev. Sci. Instrum. 73, 900 (2002) 